\title{
Confronting conceptual challenges in thermodynamics by use of self-generated analogies
}

\author{
Jesper Haglund and Fredrik Jeppsson
}

\section{Linköping University Post Print}

\section{Tweet}

N.B.: When citing this work, cite the original article.

The original publication is available at www.springerlink.com:

Jesper Haglund and Fredrik Jeppsson, Confronting conceptual challenges in thermodynamics by use of self-generated analogies, 2013, Science \&amp; Education.

http://dx.doi.org/10.1007/s11191-013-9630-5

Copyright: Springer Verlag (Germany) http://www.springerlink.com/?MUD=MP 


\title{
Confronting Conceptual Challenges in Thermodynamics by Use of Self- Generated Analogies
}

\author{
J. Haglund \& F. Jeppsson \\ Swedish National Graduate School in Science and Technology Education, Linköping University
}

\section{Introduction}

This article is part of an on-going investigation of how self-generated analogies may be used by students to express and develop their understanding of thermal phenomena in group-work settings. In previous research, we have reported on the nature of the generated analogies in group work among preservice physics teacher students (Haglund and Jeppsson, 2012). In the present study, framed within the conceptual change debate in science education research, we take a closer look on the conceptual challenges that the participants experienced during the course of the exercise. We attend to the moments where the students encounter problems in their reasoning and realise that their existing ideas and approaches are insufficient and that they will have to take another tack at the issue at hand.

\section{Theoretical background}

\subsection{Conceptual Change in Science Education}

Posner, et al. (1982) introduced the idea to describe students' learning in terms of conceptual change, in reference to the Piagetian notion of accommodation of new ideas which do not fit with a person's previously existing knowledge structure. They build their theoretical framework around a parallel between students' conceptual change in learning and revolutionary theory change in the history of science, as accounted for by Kuhn (1962) and Lakatos (1970). In particular, they propose that learners should be encouraged to abandon their previous alternative conceptions for concepts that are more in line with the current position in science. Following Lakatos, such conceptual change would be based on the conditions that the students get dissatisfied with their current understanding, e.g. when confronted by anomalies, and that the provided science concepts appear intelligible, plausible, and fruitful for getting out of their conundrum. Along these lines, Carey (1985) has contributed to the development of the so called theory theory of conceptual change, i.e. the theory that children's conceptual change is best described in terms of a shift from one theory to another, a position that has been adopted and forcefully defended in science education research by Vosniadou and colleagues (e.g. Vosniadou and Brewer, 1992) in terms of a framework theory. Chi and colleagues (e.g. Chi et al., 1994), in turn, argue that students' conceptual change often is a matter of ontological reclassification of involved concepts, such as coming to realise that heat or electric current according to the received science view are of a process nature rather than kinds of objects or substances.

The idea of individuals' conceptual change as a radical shift of theories has not remained unchallenged. Linder (1993) points to the example of grasping the wave/particle duality of physical phenomena. According to certain theories light is characterised as a wave and in others in terms of particles. Such theories provide complementary views of the physical world, which however are inconsistent with each other. This aspect is not captured in the description of learning as theory replacement. Mortimer (1995) suggests that students' 
learning of a particular taught content be characterised as a change of their conceptual profile, rather than a change of concepts, as such. Here, students could develop several parallel conceptions of a phenomenon and learning would amount to knowing in what contexts these conceptions are triggered. This adheres to the perspective of Caravita and Halldén (1994, p. 106), who argue that "the aim of learning, science for example, is not to abandon old ideas in favour of new ones, but rather to extend our repertoire of ideas about the physical and cultural world, to refine their organization and coherence”. In addition, overall, Greiffenhagen and Sherman (2008) argue that children possess nothing like the coherent structure of existing theories in science, to be replaced with other theories.

As an alternative approach to conceptual change, a resource perspective has developed in science education, in which learning is seen as a continuous development and reorganisation of previously existing knowledge rather than a radical shift. Smith et al. (1993) argue that seeing learning as a continuous process fits better than replacement approaches with a constructivist stance, in the sense that it recognised that learning is based on an individual's prior knowledge. As a central contribution within this tradition, diSessa (1993b) suggest that learning may be described in terms of a coordination of phenomenological primitives ( $p$-prims), subconscious knowledge entities, which are based on previous experiences. Other contributions to this perspective include Hammer and Elby's (2003) suggestion to make better use of students' epistemological resources in the science classroom and Sherin's (2001) study of how we interpret mathematical formulae through symbolic forms, non-formal knowledge structures. Along these lines, we have suggested that conceptual metaphors (Lakoff and Johnson, 1980) may be added to the list of productive resources for learning (Jeppsson et al., 2013).

Another strand of conceptual change research has recognised the need to complement strictly cognitive approaches to the matter with affective and motivational factors, thereby distinguishing between cold vs. warm conceptual change (Pintrich et al., 1993).

In addition, in the field of educational sciences at large, there has been a long discussion of the virtues of on the one hand cognitive approaches to learning and on the other sociocultural approaches. ${ }^{1}$ On this matter, we generally side with Sfard (1998) that the perspectives are complementary and both needed in getting a deeper understanding of teaching and learning. In particular, in line with cognitive research, we take an interest in the mind and see the individual's development of conceptions as the main target of education. However, on the other hand, we acknowledge that in the teaching situation, the teacher should take full advantage of any cultural, communicative and material resources available to achieve such learning. In line with Schoultz, et al. (2001), we believe that in order for participants to have a fair chance to show their cognitive abilities, they have to be familiar with the social setting and provided with adequate scaffolding.

\subsection{Analogies and their Use to Induce Conceptual Change}

Hesse (1966) has investigated the recruitment of analogical reasoning in scientific modelling and distinguishes between two types of analogies: On the one hand, there are formal analogies between two domains where the similarity is based on shared structural roles of the constituent entities within the domains, but not necessarily similarities of the entities across the domains. Hesse gives the example of comparing a father's children to the citizens of a state, where the father bears no evident similarities to the state. In science, formal analogies include those that are based on a shared mathematical structure, but without similarity of the corresponding entities across the domains. On the other hand, apart from the dependence on similar relational structures across domains, material analogies are also based on assumed

\footnotetext{
${ }^{1}$ See for example the debate initiated by Anderson et al. (1996) and Greeno (1997) in Educational Researcher.
} 
similarities of the involved entities across the domains. For instance, the moon is similar to the earth in being roughly spherical, celestial objects; if we know that there is no atmosphere on the moon, we might infer from our knowledge of the conditions on earth that the moon is inhospitable to humans. Applied to science, Hesse puts forward the paradigmatic case of the analogy between sound and light as wavelike phenomena, where knowledge in one domain enables predictions about the corresponding entities in the other domain; it may therefore be put to productive use in theory development. In parallel, Harré (1959) argues that models may be used as candidates-for-reality, implying a possible identity relation between a model and a phenomenon it represents, or, alternatively, in terms of coming to regard a phenomenon as similar to something else, in spite of knowing that they are categorically different.

The use of analogies has often been put forward as a helpful tool in science teaching (e.g. Aubusson et al., 2006; Duit, 1991). Gentner's (1983) structure-mapping theory has been influential in characterizing the structure of analogies, by use of the example of the Rutherford-Bohr analogy between the atom and a solar system, where knowledge about a (source) domain, here planetary systems, is mapped or transferred to a less familiar (target) domain, the atom. Gentner emphasises that analogies focus on mapping of the relational structures between entities more than on attributes of individual entities. For instance, when comparing the atom to a solar system, the focus should be on small entities (i.e. electrons and planets) revolving around a larger entity (i.e. the atomic nucleus and the sun), rather than on the fact that the sun is warm and yellow. When analogies are used in science teaching, it is typically in the form of the teacher presenting an analogy to the learners in order to introduce a new domain. However, it has also been recognised that analogies potentially are a 'doubleedged sword' (Glynn, 1989), in that learners may have difficulties drawing the same conclusions as their teachers. For instance, Gick and Holyoak (1980) found that students had difficulty applying spontaneously their experiences from solving the problem of assaulting a fortress by attacking from all angles, when asked to find suitable directions of radiation of a cancer tumour.

Since the beginning of conceptual change research, is has been recognised that analogies may facilitate students' conceptual change. Posner, et al. (1982, p. 225) argue that in teaching which is geared towards stimulating conceptual change, "[a]ny available metaphors, models, and analogies should be used to make a new conception more intelligible and plausible". Treagust et al. (1996) investigated the potential of analogical teaching in engendering conceptual change by interviewing $10^{\text {th }}$-grade students regarding the concept of refraction, the bending of light as it goes from one optic medium to another, such as air, water and glass, three months after a teaching sequence on geometrical optics. The students in one class had been introduced to refraction by use of analogy with a toy car whose path bends slightly as it moves from a surface of hard paper to a soft carpet, while the students in the parallel class were taught without analogies. While the students in the two groups scored equally on written test items, such as drawing the path of a light ray as it passes through a triangular glass prism, the class that was introduced to the analogy could articulate their understanding better than the other class, based on the criteria of intelligibility, plausibility and fruitfulness. Treagust et al. provide the alternative interpretations that the analogy either engendered a deeper understanding of the phenomenon or helped the students expressing their understanding.

Representing the resource perspective on conceptual change, Brown and Clement (1989) show, through the use of tutoring interviews about normal forces and Newton's third law, that students may be convinced to overcome their misconceptions of a situation if they are presented to a series of bridging analogies. However, interestingly, they also provide examples where students' conceptual change is not brought about. In reference to Hesse's (1966) account of material analogies and Harré's (1959) notion of candidates-for-reality, they argue that for particularly deep-seated misconceptions, such as the conviction that objects in 
uniform motion 'have force', it is often not enough to provide correspondences across domains in analogies; in addition, the chosen analogies should provide plausible explanations for the phenomena, and thereby be explanatory models.

Further, Brown (1993) argues that in order for analogies to bring about conceptual change, they often involve concrete comparisons to prior experiences and coming to attribute new properties to the entities in the target domain. For instance, in order to grasp that a table exerts a normal force on a book on top of it, students may be helped by realising the table's "springiness" at a microscopic level, rather than just seeing it as an obstacle. This focus on concrete properties stands in contrast to Gentner's (1983) emphasis on abstract relational structure. In addition, Brown proposes that in order to be useful, conscious instructional analogies should be grounded in students' subconscious core intuitions, exemplified by diSessa's (1993b) p-prims and Lakoff and Johnson's (1980) notion of an experiential gestalt of causation, found by Andersson (1986) to be used by science students in relation to a broad range of science content.

\subsection{Self-Generated Analogies as a Tool for Conceptual Understanding}

There seems to lie a paradox in that we use analogical reasoning as part of understanding in our everyday lives, but have difficulties performing in laboratory tasks, such as the one reported by Gick and Holyoak (1980). Blanchette and Dunbar (2000) propose that one way out of the paradox is to contrast teacher-generated analogies with self-generated analogies, where the learner him- or herself is asked to come up with analogies to a phenomenon. For teacher-generated analogies, the learners have to confront the double challenge of understanding the given source domain (do all children actually understand the structure and dynamics of a planetary system?), and the particular ways in which it is supposed to be similar to the unknown target domain. Similarly, Clement (1987) has studied students' use of spontaneous analogies in problem solving, without their being encouraged by teachers or researchers. In this vein, Pittman (1999) argues that the use of student-generated analogies rather than teacher-generated analogies resonates well with the constructivist assumption that learning is based on the students' prior knowledge.

While most studies on analogical reasoning in science education have been carried out in the teacher generated mode (Pittman, 1999), an increasing number of studies have adopted the approach of self-generated analogies (Haglund, 2013). For instance, Wong (1993) presented eleven preservice teacher students with a piston/cylinder device and the phenomena that the force required to pull or push the piston increases the further the gas is either compressed or decompressed when the cylinder's nozzle is covered by a finger. In addition, when released, the piston returns to the original position. Working individually, the participants were first asked to explain the experienced air pressure phenomena, evaluate their explanations and try to identify any inadequacies. Second, they were asked to generate their own analogies for the phenomena, and specify in what respects the compared domains were similar or different to each other. Third, they were asked to evaluate the analogies and modify them or come up with new ones. The participants came up with several analogies, some of which were based on microscopic ways of reasoning, such as a people-in-a-room analogy, in which the gas is compared to people mingling as in a party, or bouncing rubber balls. Other analogies were of a macroscopic character, involving comparisons to for example rubber bands and tug-of-war.

Impressive learning results and fruitful teaching approaches based on self-generated analogies have been reported in science education research. For instance, James and Scharmann (2007) achieved impressive learning gains as measured against the Force Concept Inventory (Hestenes et al., 1992) in preservice teacher education on Newton's third law by use of self-generated analogies. In chemistry teaching, Mozzer and Justi (2012) report on a 
study where 13- to 14-year-olds were asked to generate analogies for chemical bonding in clinical interviews before and after a teaching sequence on the subject. In the interviews after the teaching, the students were able to elaborate the analogies they had generated beforehand, corresponding to the acquired knowledge, such as accommodating repulsive forces between atoms in a molecule into their analogies.

More recently, the social dimension of generation and scrutiny of analogies in discourse has also received increasing attention (Haglund, 2013). For instance, Aubusson and Fogwill (2006) studied the effect of inviting secondary chemistry students to cooperate in the development of a role play as an analogy of chemical bonds, where the students acted as atoms and ions. The students expressed and negotiated their understanding in conversation as the analogy role play developed, encompassing new features gradually.

As a contrast to findings primarily in favour of self-generated analogies, Clark (2006) is cautious that with such constructivist approaches, students may spend too much time on their idiosyncratic explanations, rather than building a conception in line with the position in science. Similarly, Zook (1991) warns that education research has not acknowledged the risk of students developing misconceptions as a consequence of teaching with analogies. Yerrick, et al. (2003) studied preservice physics teachers working with analogies for electric circuits in small group exercises within a guided-inquiry approach. While strategically inserted teachergenerated analogies effectively led the students to ascertain their own beliefs, when the analogies were adapted in small-group discussions, they tended to promote misconceptions and to be over-generalised in contexts where they did not apply. In particular, self-generated analogies, corresponding to the students' personal theories were found to divert them from the focus on the studied phenomena, lead to incorrect conclusions, and unconstructive group dynamics.

\subsection{Conceptual Understanding of Thermodynamics}

The present study focuses on analogies for two processes within the thermodynamics domain of physics. Thermodynamics may broadly be described as the science of transfer and transformation of energy. The first law of thermodynamics states that the energy is conserved, that it is constant and cannot be created or destroyed but only transformed or transported. The second law of thermodynamics reflects the irreversible character of spontaneous processes: energy cannot transfer spontaneously by means of heating from a colder to a hotter system. By introduction of the physical quantity entropy, the second law of thermodynamics can be stated as: the entropy of an isolated system tends to increase and never decreases. ${ }^{2}$

In empirical studies, it has been found that university students have difficulties in grasping the concept of entropy. For instance, Sözbilir (2001) investigated the conceptual understanding in chemical thermodynamics among undergraduate chemistry students by use of problem solving exercises. One result was that only few students used the concept of microstates to explain entropy changes, while the majority saw entropy as disorder, which often led to wrong conclusions. The few students that did mention microstates were not found to have an adequate understanding of the concept. Similarly, Brosseau and Viard (1992) asked ten PhD students in physics what happened to the entropy in reversible, adiabatic expansion of an ideal gas. Only one of the students grasped that entropy is constant. Seven of the students argued that as the volume increases, so does the disorder, and hence, so does the entropy. In this way, by applying the disorder metaphor for entropy, the students encompassed the contribution of spatial configuration to the entropy alone and failed to realise the energy contribution. In previous research, we have found that a pair of physical chemistry PhD students solving problems in pairs were able to conclude that the entropy of an

\footnotetext{
${ }^{2}$ For a brief introduction of key thermodynamics concepts, please see Appendix I.
} 
ideal gas remains the same in reversible, adiabatic expansion, but were at one stage disturbed by their intuition that the entropy should increase (Jeppsson et al., 2013).

In the same vein, Carson and Watson (2002) found that undergraduate chemistry students do not use microstates or energy levels, but generally refer to disorder, when explaining entropy. Granville (1985), in turn, traces a difficulty in understanding the entropy change in adiabatic processes to a failure to grasp the importance of reversibility. In this respect, if there is adiabatic, reversible change, then the entropy is constant, but if it is irreversible, then the entropy increases. Christensen, Meltzer and Ogilvie (2009) performed an extensive study on students' conceptions of entropy and the second law of thermodynamics as expressed in problem solving regarding thermal processes. Their main finding was that the idea that the entropy of a system and its surrounding is conserved in spontaneous processes is one of the most common misunderstandings among students, for instance during free expansion of an ideal gas where the gas in a container is allowed to expand into a vacuum and the entropy actually increases. This idea may be due to confusion between entropy and energy, another extensive quantity which is conserved.

Loverude, Kautz and Heron (2002) and Leinonen, et al. (2009; 2012) have found that undergraduate physics students try to apply the ideal-gas law also for processes where crucial information is missing, such as in reversible, adiabatic expansion or compression, but fail to recognise the need to use the first law of thermodynamics in problem solving. Consequently, in his reflection on thermodynamics teaching, Cartier (2011, p. 1536) notes: "The adiabatic reversible expansion of a perfect gas is conceptually the most challenging and complex example of gas expansion to grasp and is typically the last of the expansion processes to be introduced." Students also tend to misapply microscopic models in problem solving exercises. For instance, students have been found to see collisions of particles as the cause of temperature or that energy was released as a consequence of the collisions (Leinonen et al., 2009). In parallel, Cochran and Heron (2006) have found that students have difficulties applying the second law of thermodynamics when assessing the feasibility of heat engines. Albeit so, in his constructivist spirit, Duit (1984) suggests that the second law of thermodynamics may fit better than the first law of thermodynamics with our everyday understanding of energy as being used up or wasted. Even though energy does not disappear as it is transformed or dispersed, its ability to perform work gradually decreases.

Regarding conceptions of temperature in relation to free expansion of an ideal gas, Bucy (2007) provided university students taking advanced thermodynamics courses with problem solving exercises on thermodynamic processes, including free expansion. In spite of the fact that the temperature was stated to remain constant throughout the process, some of the students declared that the temperature decreased, indicating an underlying intuition.

In fact, the constant temperature of free expansion was an important empirical finding in the development of modern thermodynamics and in the abandonment of the caloric theory of heat. In 1845, Joule (1898) reported on free expansion experiments that: "no change of temperature occurs when air is allowed to expand in such a manner as not to develop mechanical power" (p. 26, italics in original). In conjunction with other related experiments, he concluded: "These results are inexplicable if heat be a substance” (p. 29), since in such case "also a certain quantity of cold would have been produced..." (p. 29), interpreted as a temperature decrease, which did not occur. ${ }^{3}$ Joule continues: "The results are, however, such as might have been deduced a priori from any theory in which heat is regarded as a state of motion among the constituent particles of bodies" (p. 29), i.e. a mechanical theory of heat. In relation to the experiment of free expansion, he explains: "no cold was produced, because the momentum of these particles was not permanently converted into mechanical power, but had

\footnotetext{
${ }^{3}$ The rationale for this conclusion is that within the caloric theory of heat, heat in the form of caloric is assumed to be a self-repulsive fluid attracted to matter, implying a dependence on the density of the matter.
} 
the motion of the air from one vessel to the other been opposed in such a manner as to develop power at the outside of the jar, which might have been accomplished by means of a cylinder and a piston, then loss of heat would have occurred...” (p. 29). ${ }^{4}$

\subsection{Analogies in Thermodynamics}

As we have seen, use of analogy has been proposed as an approach to science teaching in general, and thermodynamics as a field is not an exception. Both formal analogies and material analogies, in Hesse's (1966) characterisation, have been used in the advancement of science as well as in the subsequent teaching of its results. Carnot (1824) made explicit use of the analogy between heat that flows from a warm object to a colder object and the water of a waterfall that flows from a higher to a lower level in his investigation of the theoretical limits of the efficiency of heat engines, within the paradigm of the caloric theory of heat. Gentner and Jeziorski (1993) put forward Carnot's comparison between heat flow and a waterfall as a typical example of the modern use of analogy with a focus on structural coherence. Such use of analogies is contrasted with the preceding use of metaphors, based on superficial symbolism and a series of associations. One example from alchemy is the association of the element iron to the colour red, the planet/god Mars, and masculinity. Particularly if applied in current teaching, where the underlying assumption of heat as caloric has been rejected, the waterfall analogy would be predominately of the formal kind, emphasising the mathematically similar structure in the two domains. In the Karlsruhe Physics Course, Herrmann (2000) emphasises the parallel between the conjugated entropy and temperature difference: $S \Delta T$, and weight and height: $m g \Delta h$, as expressions for the involved energy transformations. Here, entropy is given a substance-like interpretation, close to the everyday conception of heat as contained in warm objects. A similar approach has been adopted in engineering thermodynamics in the study of flows of matter, energy and entropy: "Now, it is suggested to the students that they can interpret entropy to be representative of what laypeople (including themselves) call 'heat' - 'heat content'” (Gaggoli, 2010, p. 1049).

A material analogy (Hesse, 1966), in contrast, lies behind Maxwell's and Boltzmann's development of the kinetic theory of gases, where the assumed molecules of an ideal gas initially were compared to inert balls that collide elastically with each other in threedimensional space, which conveyed an understanding of a hypothesised underlying microscopic mechanism. However, at the time, they were well aware that it was a crude model; molecules are nothing like elastic point particles - for instance, in parallel, Boltzmann had started to model rotational motion of molecules (Achinstein, 1987). In our interpretation, early kinetic models of ideal gases are good examples of explanatory models (Brown and Clement, 1989), yet clearly not candidates-for-reality (Harré, 1959) for the involved researchers. The kinetic theory of gases was subsequently elaborated in order to account for a wider range of phenomena, such as viscosity, but it took time for it to be generally accepted in the research community. In this way, apart from its use in building conceptual understanding, the kinetic theory of heat also provides a telling story about the nature of science, which may be fruitful in a history and philosophy of science approach to science teaching (Niaz, 2000).

\subsection{Purpose of the Research}

The purpose of the current research was to investigate what conceptual challenges preservice physics teacher students encountered when generating analogies in small-group work for two thermal processes, and how and to what degree they came to terms with these challenges. In

\footnotetext{
${ }^{4}$ The temperature decreases in free expansion of real gases, however, where attraction between particles cannot be neglected, since energy is required to separate the particles in the expansion. See e.g. Cheng (2006) for a quantitative treatment of the cooling of a van der Waals gas in free expansion.
} 
relation to a previous analysis of the generated analogies and the process of generating them (Haglund and Jeppsson, 2012), the current study is a reanalysis of the data with a particular focus on the students' conceptual challenges and the issue of conceptual change in science education research. Accordingly, the research was guided by the following research questions:

- What conceptual challenges did preservice physics teacher students confront when generating analogies for two thermodynamic processes?

- How and to what degree did they come to terms with these challenges?

- What implications do the results have for conceptual change research?

\section{Method}

We take an interest in the individual's mind and conceptions, particularly with regards to students' understanding of scientific accounts of natural phenomena and conceptual change. However, we adhere to the view of Schoultz et al. (2001) that previous research in a cognitive tradition with similar aims and interests has been carried out predominately by means of methods such as clinical interviews, multiple choice and paper-and-pencil tests, which have not reflected the full cognitive potential of the participants. Overall, we have tried to establish richer collaborative practices than what is achieved with such data collection approaches. In this respect, we have striven towards establishing an authentic environment, in the respect that the exercises are similar to what might happen in a real class-room as part of the regular education program. In particular, this study regards how eight $(\mathrm{N}=8)$ preservice physics teacher students in the fourth year of a Swedish university secondary mathematics and physics teacher education program generated their own analogies for two thermodynamic processes: reversible, adiabatic expansion of an ideal gas, and; free, adiabatic expansion of an ideal gas.

The use of self-generated analogies in a collaborative setting was seen as a productive way to take advantage of the students' prior knowledge of thermodynamics, but also in other fields that may appear as structurally similar in some way. The study thereby adheres to the view of the resource perspective on conceptual change in adding to and enriching the prior understanding in a continuous way, rather than in terms of replacement. However, in contrast to the notion of p-prims (diSessa, 1993b), which focuses on intuitive understanding within a domain, self-generated analogies, like conceptual metaphors (Jeppsson et al., 2013), rely on comparisons across domains. Furthermore, the social setting was motivated by its similarity to regular small-group exercises in the teacher education, thereby enhancing the study's ecological validity, and by providing the students with an opportunity to "talk science" (Lemke, 1990), i.e. give active contributions to dialogue with the taught subject in focus.

\subsection{Studied Phenomena}

The students were provided with the following descriptions in writing of the two processes, which incorporate both macroscopic and microscopic aspects.

Description of Process 1, Reversible, adiabatic expansion:

An ideal gas is kept in a container by a frictionless piston. The gas has amount of substance $n$, temperature $T$, internal energy $U$, pressure $p$, volume $V$ and entropy $S$. Let the gas expand slowly, adiabatically (i.e. no heat $Q$ is exchanged with the environment) and reversibly (i.e. so that the process "can go in the other way" to restore the original state) by retracting the piston. As the piston retracts, work $W$ is performed by the gas on the environment, which means that the internal energy $U$ of the gas decreases. At the same time, the volume $V$ increases and the pressure $p$ decreases. The temperature $T$ decreases. The average velocity of the gas particles decreases, because they will have a lower velocity after collisions with the withdrawing piston. The entropy does not change during the process, since no heat has been exchanged with the environment and the process is reversible, a situation represented by the following relation: $d S=$ $d Q / T$. 
Description of Process 2, Free, adiabatic expansion:

\begin{abstract}
An ideal gas is kept in one half of an insulated container separated by a thin wall. There is a vacuum in the other half. The gas has amount of substance $n$, temperature $T$, internal energy $U$, pressure $p$, volume $V$ and entropy $S$. After the instantaneous removal of the wall, the gas expands freely in the entire container. No work $W$ or heat $Q$ is exchanged with the surroundings, and hence, the internal energy remains constant. As a result, the volume of the gas is doubled and the pressure is halved. Since the particles collide with the stationary walls, their average velocity does not change. The temperature $T$ is constant, but the entropy $S$ increases.
\end{abstract}

The processes and descriptions were selected with the intention that they should be accessible to the participants, but at the same time challenging to explain. Therefore, we provided explicit accounts of what happens to the constituent particles and the involved physical quantities, but not of all relevant causal connections. For instance, the students were informed that the temperature remains unchanged in the free expansion, but it was left to them to justify why that was the case. In this respect, the approach adhered to the instructional tactic of completion problems (van Merriënboer et al., 2002), in which students are provided the answers to problems and asked to fill in gaps from the problem formulation to its solutions.

As a hypothesis, in general we expected the students to find the reversible, adiabatic process challenging to account for, as pointed out by Cartier (2011). In particular, we thought that it would be challenging for the students to account for why the entropy remains unchanged in Process 1, in spite of increased volume, due to the influence of the disorder metaphor, in line with the findings of Brosseau and Viard (1992). In Process 2, the main challenge for them was expected to be explaining why the temperature remains the same, even though the volume increases, as reported by Bucy (2007).

\title{
3.2 Data Collection
}

In the weeks prior to the study, all the participants had completed a course in mechanics and thermodynamics that included macroscopic thermodynamics and a brief introduction to particle models, including the kinetic theory of gases and a microscopic explanation of entropy in terms of disorder and microstates. However, it did not offer an in depth account of statistical mechanics including the connection between energy and microstates.

Initially, the participants were introduced to the approach of analogies and particularly self-generated analogies in teaching. Next, the participants worked in two groups consisting of four students in each, S1, S2, S3 and S4 in Group A, and S5, S6, S7 and S8 in Group B, and they sat in individual rooms. They were instructed to talk about the processes and identify what is difficult to grasp in the descriptions and to generate analogies for the processes, with a particular emphasis on entropy. The students were encouraged to come up with as many analogies as possible, and asked to select a few of them that they found particularly apt or interesting to be presented to the other group and the researchers. When the students presented their, the researchers provided the additional information that microstates are related to the distribution of energy across quantised levels, not only to spatial configuration, based on the groups' challenge with Process 1. This additional information may be considered as a kind of scaffolding (Wood et al., 1976) to the problem-solving process. Next, the groups had the opportunity to elaborate their analogies, and finally there was a sum-up and full-group discussion on the usefulness of self-generated analogies in teaching. All discussions were video recorded and the recordings fully transcribed.

\subsection{Data Analysis}

In our previous study of the student exercises, we analysed the character of the generated analogies and the process of analogical reasoning (Haglund and Jeppsson, 2012). In contrast, 
in the present study, the focus was on the conceptual challenges in relation to the scientific content at hand that the participants encountered during the exercise, and their process of coming to terms with them, arguably corresponding to conceptual change. Some of these challenges reflected underlying misconceptions or alternative conceptions, as a consequence of misunderstandings from everyday life or only partially grasping of previous teaching, but others were new or difficult situations, where they simply lacked appropriate background knowledge.

The challenges were identified by reading though the transcripts and coded by use of MAXQDA, a software for qualitative data analysis. The search for and categorisation of challenges was informed by students' misconceptions and difficulties, reported in science education research, most importantly the challenges to grasp the constant entropy in reversible, adiabatic expansion, and the constant temperature in free, adiabatic expansion.

In addition, the data are used to discuss the character of the students' conceptions of the involved natural phenomena and scientific concepts, against the background of different perspectives on the issue of conceptual change, as presented in science education research. In particular, we bring up the implications of the students' use of a wide range of source domains in generating analogies for the two processes.

\section{Results}

An overall finding of the study is that the students came up with several analogies, some of which were generated by themselves and others recalled from prior teaching. In our previous analysis (Haglund and Jeppsson, 2012), the self-generated analogies were found to be developed to a greater structural depth (Gentner, 1983), in comparison to the teachergenerated analogies. In contrast to previous research (Blanchette and Dunbar, 2000), we did not primarily attribute this difference to cognitive factors, but saw it as an expression of the students assuming ownership (Enghag and Niedderer, 2008) for their own analogies. For instance, one of the groups adopted a protective stance towards an analogy that they selected for presentation to the other group and us as researchers, and argued that it would be up to us to identify any of its shortcomings, indicating an affective dimension to the exercise.

The groups produced about twenty self-generated analogies, all of which, intriguingly, were based on a microscopic perspective, where objects such as bouncing balls, angry bees and people mingling at a party corresponded to particles of a thermodynamic system (Haglund and Jeppsson, 2012). Such microscopic accounts of the encountered phenomena share with the notions of notions of material analogies (Hesse, 1966) and explanatory models (Brown and Clement, 1989) the characteristic of providing an underlying mechanism in order to explain the phenomena. However, the students run no risk of conceptualising the bees, people, etc., as identical to of the same kind as atoms or molecules, which shifts such analogies towards the formal analogy (Hesse, 1966) category slightly and exclude them as candidates-for-reality (Harré, 1959). In contrast, when students adopt billiard ball explanations from prior teaching, it is possibly a tempting idea that microscopic particles actually are inert hard-spheres, hence candidates-for-reality.

In all, 23 different challenges were identified in the transcripts, including the two challenges inherent in the task design, i.e. accounting for the constant entropy in adiabatic reversible expansion and accounting for the constant temperature in free expansion. In the sections below, we choose to bring up the six challenges to which the students dedicated the largest number of utterances in their discussions, and were identified in the discussions of both groups:

- The meaning of entropy

- The meaning of microstates 
- Constant entropy in adiabatic expansion of an ideal gas

- Connection between entropy and microstates

- Constant temperature in free expansion of an ideal gas

- Consequence of collisions of particles

The way in which the students encountered these challenges and came to terms with most of them is presented below in two sections, one with a focus on conceptions of entropy corresponding to the first four challenges, and the other with a focus on conceptions of temperature, corresponding to the last two challenges. The challenges are presented in the form of example excerpts, framed in the contexts in which the dialogues took place, and analyses of what happens.

\subsection{Students' Conceptual Challenges regarding Entropy}

When the students in both groups tried to explain the entropy change in the two processes, they expressed explicitly that they did not know what entropy really is or only had a vague idea of the concept, reflecting the general challenge of grasping 'the meaning of entropy'. For instance, as mentioned, Group B introduced the analogy between a thermodynamic system and angry bees in a jar, where the "angriness" of the bees corresponded either to the temperature or the energy of the system or its constituting entities. They used the analogy in accounting for the reversible adiabatic expansion, but had difficulties incorporating the constant entropy:

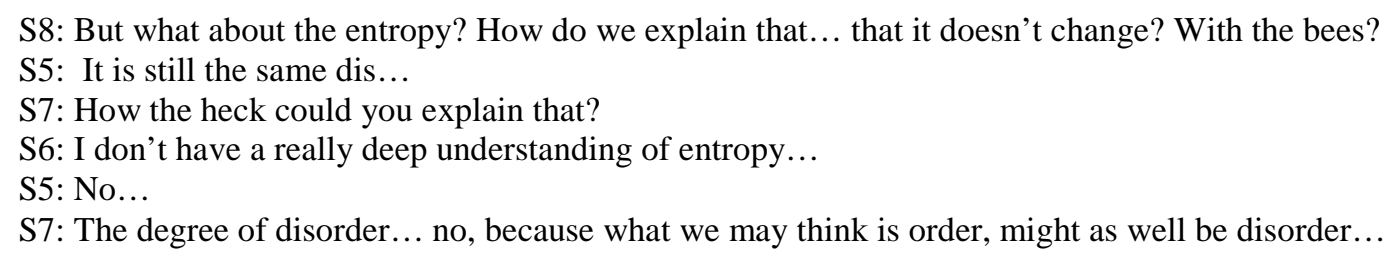

In this excerpt, we see that the students used the metaphor 'entropy is disorder', but also realised its subjective character which reflects the view that it is difficult to apply disorder in problem solving situations. There is also a sign of confusion and frustration in not being able to understand why the entropy is constant in this particular process; it seems to contradict their intuition. Hence, as expected there is a challenge for the students in accounting for the constant entropy in adiabatic expansion of an ideal gas.

Even though the students had not taken a course in statistical mechanics, they spontaneously used the concept of microstates in an apparently confident way. However, they tended to recognise only the configurational aspect, which relates microstates to the volume of a system, but ignored the energy contribution to the microstates, reflecting another challenge: 'the meaning of microstates'. For example, during discussions regarding the constant entropy in Process 1, S3 in Group A said: "Well... the microstates and the volume... they are pretty much the same thing." This focus on the volume is assumed in spite of the example of formation of ice being used in the previous thermodynamics course in order to introduce the energy contribution to entropy and showing that visual spatial configuration does not tell the entire story. Individual students in both groups mentioned the need to consider the energy contributions to the entropy, but somehow this aspect was not incorporated spontaneously into any of their analogies of the phenomena.

The students knew from their previous teaching that entropy is strongly connected to microstates. However, as mentioned above, the configurational emphasis on microstates made it difficult to account for the constant entropy in the reversible, adiabatic expansion. Therefore, there was another challenge in understanding the specific character of the 
'connection between entropy and microstates'. An initial, not clearly expressed idea was that the constant entropy had to do with the reduced energy, temperature or velocity in some way. For example, in the group exercise, S6 related the constant entropy to the reduced 'angriness' of the bees corresponding to the temperature of the system. Similarly, in the Group A, S3 thought that the velocity would be involved:

S4: The entropy 'S' doesn't change during the [reversible, adiabatic] process.

S3: But you have greater volume there [when the piston has withdrawn] where they [the particles] can be located... it has to do with the velocity and the volume...

In our view, these vague ideas have an inbuilt potential, which could have been developed into accounts in line with views sanctioned in science. However, the vague connections between entropy/microstates and velocity/temperature/energy evolved independently in both groups into the hypothesis that the number of microstates increases in the adiabatic expansion, but that the entropy remains constant. The hypothetical idiosyncratic explanation they developed was that the entropy is not a function of the number of microstates itself, according to Boltzmann's microscopic interpretation of entropy as $S=k_{B} \ln \Omega$, but a function of the changes of microstates. In their view, since the particles are slower and further apart when the volume has increased, it takes longer time to shift between microstates. In the joint presentation to the researchers, members from both groups expressed:

S2: I don’t know, maybe I understood entropy a bit better... If there are more microstates, you could say that they [the particles] change places more often. But if they have a lower velocity...

S6: But we have just as many microstates, don't we?

S7: OK, you get more...

S2: ...microstates, but you have a lower velocity, so it's rather change of place that is the entropy, then.

Now, after the initial round of generation of analogies and presentations to the researchers and the other group, the researchers brought to attention the concept of energy levels and the influence of energy decrease on the number of microstates, in order to give an alternative to the idiosyncratic explanations that the students had given. In the following group exercise where the analogies were developed further, the information on the role of energy levels helped the students elaborate their understanding of the connections between entropy and microstates. By the introduction of energy levels, the groups expressed less frustration regarding the constant entropy in reversible adiabatic expansion. For instance, Group B started by incorporating the new view on entropy into their 'angry bees' analogy, where the angriness of individual bees corresponds to energy levels:

S6: We would have to start by saying that the entropy depends on... on one hand, how many...

S8: ... how many locations the bees can be at...

S6: Yes. And on the other hand...

S8: ... on the other, how angry they are...

S5: Yes.

S7: ... what level of angriness they are at...

However, not all students had yet developed a fully coherent account of the phenomenon. For example, the idiosyncratic explanation that entropy is connected to the change between microstates still lingered in parallel with accounts more in line with the position in science:

S1: But what do you think the entropy is, then? /.../

S2: I think you can see the entropy more like the change... /.../ It's like the number of changes of places per time unit that corresponds to the entropy. 
S3: But it wasn't like that [according to the researchers]. It was a matter of losing energy, microstates... well, the energy microstates... you increase the microstates that consider the volume, but lose the ones that consider the energy.

S2: Yes, because it goes too slow.

S3: Yes, the net entropy [change] is zero.

S3 had adopted the perspective of the number of microstates being related to energy levels, which may be interpreted as having expanded his repertoire of how to conceptualise thermal processes. In contrast, S2 stuck to the idiosyncratic idea of entropy being a function of the rate of change between microstates and, due to the fact that he associated straight from decreased energy to decreased velocity, does not seem to have grasped that the two explanations are different and cannot be combined in a coherent way. In addition, as indicated below when relating to Process 1, S3's previously exposed conception of microstates as spatial configuration is not replaced. Instead, a new meaning of microstates has been added to the former:

S1: Ok, but what is the entropy...? /.../

S3: It is the microstates of the energies and the microstates.

In the last verbal output above, these two meanings or conceptions of the word 'microstates' seem to be used in the very same sentence! This may be interpreted as in the second instance, it is the earlier interpretation of spatial configuration only, and in the first, it is the compounded interpretation comprising spatial configuration and distribution across energy levels.

Finally, at the closing debriefing discussion, the students expressed that they had developed their understanding somewhat during the exercise, but entropy was still perceived as a vague concept, as exemplified in the following excerpt:

S6: Well, we have learnt a bit about entropy. [laughter]

S2: Yes, it reinforces a bit more what entropy is... /.../ That it is something very intangible... it's just like a measure of something... but you have understood this 'something' a little bit better...

\subsection{Students' Conceptual Challenges regarding Temperature}

As expected, both groups found it challenging to account for the 'constant temperature in free expansion of an ideal gas'. For example, in the initial group discussion, S5, S6 and S7 said:

S7: The temperature will decrease here [during free expansion]

S5: No.

S7: [Reading the task] 'The temperature is constant' /.../

S5: It's just that, if the volume increases, then the pressure decreases.

S7: Yes, but then the temperature decreases, as well.

S5: But it [the gas] is so isolated, so the temperature.../.../ you don't do any work.

S7: No. /.../ But it feels kind of fuzzy all this... /.../ I still think that if you increase the volume, then the temperature should drop...

S6: Yes, but this is an isothermal [process]... Or, forget that...

S7: Yes, but in an isothermal [process], you add heat.

S6: Yes, you do... that's odd... how the heck can the temperature be constant, then... this is really weird!

The students' intuition was that the temperature should decrease with increasing volume in the free expansion and they express frustration towards the stated fact that the temperature is constant. This intuition may be due to application of the ideal gas law to the process, even though there is not sufficient information to adopt that approach. Another influence is the experience of problem solving tasks related to reversible processes, such as isothermal expansion, which they explicitly refer to. For an isothermal reversible, expansion, heat has to 
be added in order to keep the temperature constant, and for a reversible, adiabatic expansion, Process 1, the temperature decreases.

As the groups grappled with the problem, however, they realised that these macroscopic approaches were not successful. Instead, both groups turned to different idealised microscopic models, where the velocity of particles remains unchanged at the removal of a wall, and therefore, that the kinetic energy and temperature also remain constant. For instance, Group B used an analogy between the thermodynamic system and balls bouncing in free space, where they would not have to consider friction or gravity, to explain the constant temperature:

S7: A corridor in the middle of space... you throw balls that bounce like this: 'ding', 'ding' [bouncing between two walls]... and then like, 'poh' [one wall disappears] /.../ Then [the balls] will go like this instead [gestures that the balls go further and collide with another wall] at the same velocity. / .../

S6: But if the temperature is constant, of course they [the balls] have the same velocity.

As mentioned, both groups viewed thermodynamic variables such as temperature and pressure as being related to a microscopic model of collisions between particles. Specifically, as reported previously (Haglund and Jeppsson, 2012), Group B that explored the 'angry bees' analogy expressed the idea that collisions are required to maintain the "angriness" of the bees, corresponding to the temperature of the system in this account, i.e. a challenge regarding the 'consequence of collisions of particles':

S6: They [the bees] get angry if they meet with each other. Or rather... that's what keeps them angry.

S8: Yes, right.

S6: Because, they don't get angry, but they are kept angry, when they meet with each other... a constant angriness [laughter] /.../ Otherwise, you might think that they would calm down after a while, but they don't.

S7: Constantly angry because of collisions. /.../

S6: It's like an isoanger [laughter]

Through the use of this particular analogy, an anthropomorphic way of reasoning is used comparing dead objects to humans - where the angriness, corresponding to the temperature, would fade away without external interaction.

\section{Discussion}

\subsection{Discussion of Results}

Sözbilir (2001) and Brosseau and Viard (1992) have provided empirical evidence demonstrating that by use of the metaphor 'entropy is disorder', students may reach erroneous conclusions during problem solving in thermodynamics. In a similar fashion, in our study we found that the students intuitively thought that the entropy should increase in reversible adiabatic expansion in Process 1, by relying on the disorder metaphor. Against the background of the findings of Sözbilir that only a very small percentage of students use microstates in their explanations of entropy changes, one might have expected that the introduction of this concept would have been the key to sorting out this matter. However, as opposed to the findings of Carson and Watson (2002) and Sözbilir (2001), we discovered that the students in our study spontaneously used the concept of microstates, but initially with an exclusively configurational interpretation. In this way, our data show that the introduction of microstates in itself does not always automatically lead to students grasping the energy contribution to entropy. In reference to Chi et al.'s (1994) notion of ontological classifications, Brookes and Etkina (2007) argue that students run the risk of interpreting metaphors in modern physics related to locations too literally, giving the example of electrons being in a "potential well" or at a low "energy level". We see a clear parallel with the spatial 
interpretation of microstates as locations for particles to be situated in, and the challenge may be inherited in subsequent statistical mechanics teaching, where it might be tempting to identify the $6 N$ dimensional phase space with Newtonian 3D space.

In addition, the students used microscopic approaches to try to account for the entropy change in the reversible adiabatic process, and ignored the possibilities of relations from macroscopic thermodynamics. In this respect, there is a contrast to Wong's (1993) study, where undergraduate students created both macroscopic and microscopic analogies for related air pressure phenomena, some of which were similar to the ones reported in our study, such as mingling people at a party and bouncing balls. The constant entropy of adiabatic, reversible expansion is a genuinely difficult issue from a microscopic point of view. Even if the students had realised that the decreased energy provides a negative contribution to the entropy, it is far from evident that this contribution cancels the contribution from the increased volume completely. With the introduction of the notion of energy levels from the researchers, the students started to make sense of the constant entropy in the reversible adiabatic expansion. At least, by grasping that the contribution to the entropy of the increased volume is counteracted by the reduced energy of the system, constant entropy was not unimaginable any more. Here, the students traded off one component of entropy change against another; this cognitive resource has previously been described as a symbolic form (Sherin, 2001), by the name of different amounts, when it was used by $\mathrm{PhD}$ students in problem solving exercises (Jeppsson et al., 2013).

In contrast, adopting a macroscopic approach with employment of the reversible assumption and the formula $d S=d Q / T$, which was provided in the process description given to the students, would have given a more straightforward solution, but this approach was not adopted. Therefore, in addition to being hesitant to apply the first law of thermodynamics, as shown by Loverude et al. (2002) and Leinonen et al. (2009), in the current study, the students were also reluctant to use the second law of thermodynamics, a result in line with the findings of Cochran and Heron (2006). This avoidance of macroscopic thermodynamics may be due to the fact that it rarely provides causal mechanisms, the trademark of explanatory models (Brown and Clement, 1989).

As for the free, adiabatic expansion in Process 2, in line with Bucy's (2007) findings, the students intuited that the temperature should decrease. This is an interesting case of where the intuition from everyday reasoning differs from the current position in science (Joule, 1898), but incidentally coincides with the abandoned caloric theory of heat. We do not know the source of this intuition, but it may relate in some way to our experience of being close to a heat source; the closer you are to a burning fire the warmer you feel. Andersson (1986) sees this intuition as an example of a more general pattern within the experiential gestalt of causation: the nearer, the greater the effect. Applied to the current situation, it could be rephrased as: the larger the volume, the colder it gets. However, as opposed to the layperson's typical way of conceptualising temperature on a vertical scale, in relation to a spatially confined heat source (Amin, 2001), in this case there is no such heat source, except perhaps the warmth of the other people in a crowd.

The conceptualisation may also be traced to an intuitive understanding of intensity or 'spread-outness'. Knowing that the pressure of the system - an intensive quantity - decreases in the expansion, may lead to the thought that all intensive quantities, including temperature, decrease. This line of thought is often useful in problem solving involving the change of temperature in irreversible thermal processes. Dispersal of particles and energy typically implies that the system in focus loses energy to its surrounding, and thereby a decreased temperature, in line with the intuition of energy waste connected to the second law of thermodynamics (Duit, 1984). It just does not apply to the free expansion situation. The conception of decreased intensity as objects spread out reminisces of diSessa's (1993b) p- 
prim dying away, referring to the idea that all moving objects slow down and eventually come to rest, even though this p-prim has been applied to the motion of individual objects, rather than to dispersal of several objects.

In addition, the students' challenges with Process 2 may relate to previous experience of problem solving on reversible processes. When dealing with the unchanged temperature in the irreversible free expansion of an ideal gas, the students were initially influenced by their previous experience of reversible isothermal expansion. However, as opposed to Granville's (1985) finding, they realised that this irreversible process is different from the reversible processes, and thus, needs to be handled differently. Next, they tried to apply the ideal-gas law, following the findings of Loverude et al. (2002) and Leinonen et al. (2009), but again concluded that it was inadequate. The eventual successful route was to change to an idealised microscopic model of the system, by use of which they realised that the average velocity of the particles remained unchanged, and therefore, also the temperature. Here, by reference to a material analogy (Hesse, 1966) or explanatory model (Brown and Clement, 1989) which was very similar to the kinetic theory of gas, the students managed to refocus from the increasing volume of the system to the constant velocity of the involved particles. In this frame of mind, the constant temperature appeared self-evident and in line with their intuition.

However, none of the groups tried the alternative route to the free expansion process of applying the first law of thermodynamics directly and the relation between the constant internal energy and temperature, given the ideal gas assumption. This is yet another example of the students choosing not to apply macroscopic lines of reasoning.

As Clark (2006) argues, students need guidance in order not to focus too much on their idiosyncratic explanations when trying to reconcile their previous understanding and new experiences. In the current study, a case in point is the anthropomorphic idea that collisions are required for angry bees to remain angry. This idea is transferred to the domain of thermodynamics where the students argue that particles have to collide to keep the temperature up. This view on the role of collisions is similar to what Leinonen et al. (2009) found, and may offer a tentative explanation for its origin, i.e. an anthropomorphic analogy. The idea that collisions are required to maintain the temperature may also be seen as a parallel to the view that an external force is required to maintain the velocity of an object. This is a misconception commonly found in relation to the teaching of Newtonian mechanics and similar to pre-Newtonian theories, such as the medieval impetus theory or Aristotelian explanations (Clement, 1982), once again reminiscing of the p-prim dying away (diSessa, 1993b). Here, a teacher would have to intervene and point out that the analogy is taken too far. At some times, the participants can assume the responsibility of sorting the matter out themselves, but at other times, teacher interventions are necessary in order to avoid misleading idiosyncratic explanations.

Another case of an idiosyncratic explanation is the hypothesis put forward independently by both groups that entropy is a function of the change of microstates, not of the number of microstates itself. From the point of view of the groups, this seemed as an adequate way out of a dilemma. As part of the exercise, the groups put forward the idea of linking entropy to movement of particles, which they probably had not entertained before. In this particular situation, the concept of energy levels was the missing piece in the puzzle, and it had to be provided by the researchers in order for the groups to gain an understanding more in line with the current scientific position.

\subsection{Implications for Education and Research on Analogies and Conceptual Change}

We found the approach of asking groups of participants to provide their own analogies for a physical phenomenon to be a useful tool for identifying cognitive challenges to learning and understanding. Note, however, that - in line with Heywood and Parker (1997) - it is the 
process of generating and scrutinising one's own analogies that we found powerful, not the quality of the analogies as such. Instead, the task of generating analogies induced the students to 'talk science' (Lemke, 1990) and thereby come to express and negotiate their ideas. Consistent with the ideas put forward by Treagust et al. (1996), analogies helped the students put words to their understanding. In addition, as they grappled with their challenges, the students were found to ground their accounts of the phenomena and the analogies in different kinds of cognitive resources or core intuitions (Brown, 1993), including p-prims (diSessa, 1993b) and symbolic forms (Sherin, 2001).

In addition, a particularly promising result of the exercise was that the groups by themselves or with brief input from the researchers managed to make sense of and resolve most of the encountered challenges. Albeit so, we are still intrigued by the students' predominant use of microscopic ways of reasoning, which in the case of the reversible, adiabatic expansion stood in the way for a more appropriate macroscopic approach. This preference of using microscopic approaches to problem solving was mirrored by the exclusively microscopic analogies that the groups created. From one perspective, this lends support to Reif (1999), who argues in support of microscopic introductions to thermodynamics, since it is difficult for students to visualise macroscopic thermodynamics. It also reinforces the view of Brown and Clement (1989) on students' reliance on causal mechanisms in explanatory models in order to induce conceptual change. However, as pointed out by Baierlein (1994), students should be encouraged to appropriate both macroscopic and microscopic accounts of thermal phenomena and ultimately come to grasp how they relate to one another. Even though causal explanations are attractive for learning, they may also restrict creative processes. Therefore, rather than speculating on causal mechanisms of quantum phenomena and how they relate to everyday language and everyday phenomena, many modern physicists have come to adopt a "shut-up and calculate" attitude to their field (Tegmark, 1998). One possible extension of the research could be to investigate the effect of formulating the tasks in exclusively microscopic vs. macroscopic language.

As pointed out by Clark (2006), Zook (1991) and Yerrick et al. (2003), students left to themselves in generating analogies tend to develop idiosyncratic explanations and run the risk of reinforcing misconceptions. This was confirmed in the present study, where the two groups developed accounts of the phenomena which were not in line with the current position in science and most likely not provided in their previous teaching. Here, the students applied their core intuitions in ways that misled them in grasping the phenomena. However, with a brief intervention by the researchers, providing the information that microstates relate not only to the spatial distribution of particles, but also to the distribution of energy across discrete energy levels, the groups managed to adjust their accounts of the phenomena and include the energetic contribution to the microstates in their analogies. This elaboration reminds of the way the students in the study by Mozzer and Justi (2012) managed to build more components into their analogies, corresponding to their increased conceptual understanding of bonding.

How does the approach of using self-generated analogies in a collaborative setting and the findings from the present study fit within conceptual change research in science education? First, overall, we would like to point out the parallelism between self-generated analogies and the resource perspective on conceptual change in the respect that they explicitly subscribe to the constructivist idea that learning has to build on the individual's prior knowledge, as pointed out by Pittman (1999) and Smith, et al. (1993). In particular, we would argue that students' capacity to gradually elaborate their own analogies, in resolution of conceptual challenges as reported in the present study, and in correspondence with their increased knowledge as found by Mozzer and Justi (2012), also speak in favour of the resource perspective. 
Next, in our study there is no evidence of radical replacement of theories or concepts on the part of the participant student. This does not imply that such radical change cannot occur, but may add to the view that it is an unusual occurrence in individuals' conceptual development. Instead, we found support of the view of learning as coming to extend the repertoire of ways of seeing a phenomenon (Caravita and Halldén, 1994), as the students generated and explored a broad range of analogies in interaction with the researchers. As we have seen, all analogies relied on microscopic lines of reasoning, and it could be argued that they all reflected one single theory-like framework of the phenomena (Vosniadou and Brewer, 1992). However, the students made no attempts to merge their analogies into a coherent whole, and we would claim that this fragmented character of the accounts fits better within the resource perspective.

With regard to Chi's account of conceptual change as ontological reclassification (e.g. Chi et al., 1994), diSessa (1993a, p. 274) brings forward entropy as a particular challenging case, and tries to categorise it in her classification scheme:

Entropy itself does not belong to any obvious naive ontology. It is not material, or a process, or a mental state. It is not an acausal interaction, because, in fact, it is no interaction at all. /.../ And it is not constant, uniform and simultaneous everywhere, static, ongoing, equilibrium, or transfer. I believe entropy itself is the key conceptual difficulty in the second law, but Chi and Slotta have no way to describe difficulty in learning this concept.

We agree with diSessa that it appears difficult to categorise certain abstract concepts, such as entropy, in Chi's ontological classification scheme. Therefore, it is also difficult to describe learning of such concepts as a matter of clear-cut reclassification. In order to gather understanding of such complex, abstract notions, it may be necessary to go beyond ontological commitments along the lines of candidates-for-reality (Harré, 1959), and embrace many parallel conceptions in a flexible manner (e.g. Gupta et al., 2010). As we have seen, entropy may be treated metaphorically as if it has a substance-like character (Herrmann, 2000), even though we know that it is not the case literally. Even though it may be used productively in teaching, this conception is not without its flaws. It may even reinforce misconceptions, such as the idea that entropy is a conserved quantity (Christensen et al., 2009). In order to avoid the idiosyncratic character of each individual analogy, Spiro et al. (1989) suggest the use of a coherent set of multiple analogies in the teaching of complex subject matter, in their case muscle physiology. In a similar way, we think that the metaphorical use of entropy as a kind of substance could be complemented in teaching with other metaphors that bring to the fore other aspects of entropy, including entropy as 'information', 'freedom', 'spreading', or - as adopted by the students in the present study 'disorder' (see Jeppsson et al., 2011, for further discussion of these metaphors). However, in contrast to the multiple analogy approach, but as a parallel to Linder's (1993) argument with regards to the wave/particle duality, these metaphors are complementary, but most likely mutually incompatible.

Then again, we recognise that processes that are categorised as based on constraintbased interaction (Chi et al., 1994), also described in terms of emergent processes (Chi, 2005), such as microscopic accounts of diffusion, heat conduction and the electric circuit, share a common underlying structure and are challenging for students to grasp. Bringing students to see the parallel between such phenomena by encouraging analogical reasoning may be a teaching approach worth considering (Haglund, 2013).

Our finding that the students assumed ownership for their own analogies and that this contributed to their elaboration of the analogies to a great structural depth points to the importance of acknowledging motivational and affective factors, in line with warm conceptual change (Pintrich et al., 1993). In the exercises, the students also expressed 
frustration when reading that the entropy remains unchanged in reversible adiabatic expansion and that the temperature remains unchanged in free adiabatic expansion. This may be interpreted in terms of the students experiencing a cognitive conflict between their previous understanding and the taught theory, which according to Posner et al. (1982) is a promising instructional tactic for conceptual change. However, as we have seen, such emphasis on the differences between students' prior knowledge and the content to be learnt has also been criticised from, among others, proponents of the resource perspective. In our study, we think that the tactic of using completion problems (van Merriënboer et al., 2002) helped to strike a balance between pointing out a gap in the students' reasoning, which stimulated interest, and guiding them to close the gap by use of their existing cognitive resources.

In addition, in line with Schoultz et al. (2001), we would like to reiterate the potential in making full use of social interaction in bringing about conceptual change and as an approach to data collection in science education research.

\subsection{Conclusions}

We end the discussion by returning to the research questions of the study. The first research question was: What conceptual challenges did preservice physics teacher students confront when generating analogies for two thermodynamic processes? As a result of the study, the students met with conceptual challenges that were, to a large degree, in line with our expectations and previous empirical studies on students' conceptions in the field of thermodynamics. In particular, they found it challenging to account for constant entropy in reversible, adiabatic expansion of an ideal gas, and constant temperature in free, adiabatic expansion of an ideal gas. In addition, they expressed the idea that collisions are required for molecules in a gas to have constant energy. We argue that these challenges were grounded in the students' intuitive understanding of the two processes.

The second research question was: How and to what degree did they come to terms with these challenges? Regarding the constant entropy in reversible, adiabatic expansion, the students in the two groups independently developed the idiosyncratic idea that entropy is a function of the change of microstates, and not, as according to Boltzmann's microscopic interpretation of entropy, a function of the number of microstates. However, after having been introduced to the idea of discrete energy levels and the dependence of the entropy of a system on its energy, a kind of scaffolding from the researchers, they managed to make sense of the constant entropy in a way more in line with the received position in science. As for the constant temperature in free, adiabatic expansion, the students initially intuited that the temperature should decrease, by reference to their experience of reversible processes, dispersion, and the ideal-gas law. However, in contrast to the reversible, adiabatic expansion, eventually they managed by themselves to account for the constant temperature, by using microscopic explanatory models of the phenomenon.

Finally, the third research question was: What implications do the results have for conceptual change research? Overall, the plethora of analogies generated, from different source domains, speaks in favour of the idea of students' conceptions as fragmented in character. Such views are brought to the fore within the resource perspective on conceptual change, and encompassed in the descriptions of learning as a matter of expanding the repertoire of ways to interpret physical phenomena and conceptual profile change. On the other hand, we acknowledge that it is not unreasonable to interpret the students' exclusive use of microscopic approaches to the matter as an expression of an underlying coherent thought structure. All in all, we found a continuity perspective on conceptual change with a focus on students' reliance on intuitive cognitive resources to be a powerful way to characterise both the students' challenges and how they came to terms with them. 


\section{Appendix I: Key Thermodynamics Concepts}

Introductory thermodynamics typically involves the study of different thermodynamic processes, where the state of a system, such as a box containing gas, changes. The system can exchange energy with the surrounding environment through the mechanisms of work, $W$, and heat, $Q$. Work refers to changes in macroscopic variables of the system, such as its volume, $V$, or amount of particles, $N$, and heat is typically due to a difference in temperature, $T$, between the system and its environment. The first law of thermodynamics may be stated in terms of: $\Delta U=W+Q$, i.e. that the change of internal energy of a system is equal to the work and heat added to it.

Different thermodynamic processes are characterised by the mechanisms of energy exchange and changes to involved physical quantities. For instance, the present study focuses on the processes of reversible, adiabatic expansion and free, adiabatic expansion of an ideal gas. An ideal gas is assumed to consist of randomly-moving particles that interact exclusively through exchange of energy during collisions and occupy a negligible part of the system's volume. Reversible processes are processes that can run backwards in time, while free expansion refers to allowing the gas to expand into a part of the volume that previously was inaccessible, and adiabatic means that no heat is exchanged with the environment. Equations of state, such as the ideal-gas law: $p V=n R T$, provide important information on the relation between the involved quantities, here, in addition to the temperature, the pressure, $p$, the volume, $V$, and the amount of substance, $n$, while $R$ is the universal gas constant.

Entropy, $S$, is an extensive - i.e. depending on the size of a system - physical quantity, which was introduced as a macroscopic variable, through the relation: $d S \geq d Q / T$, by Clausius, in order to account for equivalence values of work and heat in heat engines. Subsequently, Boltzmann gave entropy a microscopic interpretation in relation to the number of ways an isolated system's energy and its constituent particles can be distributed, i.e. the number of microstates, $\Omega$, according to: $S=k_{B} \ln \Omega$, where $k_{B}$ is Boltzmann's constant. Subsequently, Gibbs introduced a microscopic interpretation of entropy in relation to the probability $p_{i}$ of the system being in microstate $i: S=-k_{B} \sum_{i} p_{i} \ln p_{i}$, which could be generalised to systems that are allowed to exchange energy and particles with its surroundings.

Temperature, in turn, is an intensive quantity; if a system doubles in size, all other things equal, its temperature remains the same. Within the field of the kinetic theory of gases, a system's temperature is often introduced as proportional to the average kinetic energy of its constituent particles. However, a more foundational definition depends on the system's entropy:

$$
\frac{1}{T}=\left(\frac{\partial S}{\partial U}\right)_{V, N},
$$

i.e. the temperature is the inverse of the partial derivative of the entropy with regards to the internal energy, given constant volume $V$ and number of particles $N$.

\section{References}

Achinstein, P. (1987). Scientific discovery and Maxwell's kinetic theory. Philosophy of Science, 54(3), 409-434.

Amin, T. G. (2001). A cognitive linguistics approach to the layperson's understanding of thermal phenomena. In A. Cienki, B. Luka, \& M. Smith (Eds.), Conceptual and discourse factors in linguistic structure (pp. 27-44). Stanford, CA: CSLI Publications.

Anderson, J. R., Reder, L. M., \& Simon, H. A. (1996). Situated learning and education. Educational Researcher, 25(4), 5-11. 
Andersson, B. (1986). The experiential gestalt of causation: a common core to pupils' preconceptions in science. European Journal of Science Education, 8(2), 155-171.

Aubusson, P. J., \& Fogwill, S. (2006). Role play as analogical modelling in science. In P. J. Aubusson, A. G. Harrison, \& S. M. Ritchie (Eds.), Metaphor and analogy in science education (pp. 93-104). Dordrecht, the Netherlands: Springer.

Aubusson, P. J., Harrison, A. G., \& Ritchie, S. M. (Eds.). (2006). Metaphor and analogy in science education. Dordrecht, the Netherlands: Springer.

Baierlein, R. (1994). Entropy and the second law: A pedagogical alternative. American Journal of Physics, 62(1), 15-26.

Blanchette, I., \& Dunbar, K. (2000). How analogies are generated: The roles of structural and superficial similarity. Memory \& Cognition, 28(1), 108-124.

Brookes, D. T., \& Etkina, E. (2007). Using conceptual metaphor and functional grammar to explore how language used in physics affects student learning. Physical Review Special Topics - Physics Education Research, 3(1), 010105.

Brosseau, C., \& Viard, J. (1992). Quelques réflexions sur le concept d'entropie issues d'un enseignement de thermodynamique (Some reflections on the entropy concept from thermodynamics teaching). Enseñanza de las ciencias, 10(1), 13-16.

Brown, D. E. (1993). Refocusing core intuitions: A concretizing role for analogy in conceptual change. Journal of Research in Science Teaching, 30(10), 1273-1290.

Brown, D. E., \& Clement, J. (1989). Overcoming misconceptions via analogical reasoning: abstract transfer versus explanatory model construction. Instructional Science, 18(4), 237-261.

Bucy, B. R. (2007). Investigations of student understanding of entropy and mixed secondorder partial derivatives in upper-level thermodynamics. Doctoral Dissertation, University of Maine, Orono, ME.

Caravita, S., \& Halldén, O. (1994). Re-framing the problem of conceptual change. Learning and Instruction, 4(1), 89-111.

Carey, S. (1985). Conceptual change in childhood. Cambridge, MA: MIT Press.

Carnot, S. (1824). Réflexions sur la puissance motrice du feu er sur les machine propre à développer cette puissance (Reflections on the motive power of fire). Paris, France: Bachelier.hej

Carson, E. M., \& Watson, J. R. (2002). Undergraduate students' understandings of entropy and Gibbs' free energy. University Chemistry Education, 6(1), 4-12.

Cartier, S. F. (2011). The statistical interpretation of classical thermodynamic heating and expansion processes. Journal of Chemical Education, 88(11), 1531-1537.

Cheng, Y.-C. (2006). Macroscopic and statistical thermodynamics. Hackensack, NJ: World Scientific Publishing.

Chi, M. T. H. (2005). Commonsense conceptions of emergent processes: Why some misconceptions are robust. Journal of the Learning Sciences, 14(2), 161-199.

Chi, M. T. H., Slotta, J. D., \& De Leeuw, N. (1994). From things to processes: A theory of conceptual change for learning science concepts. Learning and Instruction, 4(1), 2743.

Christensen, W. M., Meltzer, D. E., \& Ogilvie, C. A. (2009). Student ideas regarding entropy and the second law of thermodynamics in an introductory physics course. American Journal of Physics, 77(10), 907-917.

Clark, D. (2006). Longitudinal conceptual change in students' understanding of thermal equilibrium: An examination of the process of conceptual restructuring. Cognition and Instruction, 24(4), 467-563.

Clement, J. (1982). Students' preconceptions in introductory mechanics. American Journal of Physics, 50(1), 66-71. 
Clement, J. (1987). Generation of spontaneous analogies by students solving science problems. Paper presented at the International Conference on Thinking (3rd), Honolulu, HI, January 4-8, Honolulu, HI,

Cochran, M. J., \& Heron, P. R. L. (2006). Development and assessment of research-based tutorials on heat engines and the second law of thermodynamics. American Journal of Physics, 74(8), 734-741.

diSessa, A. A. (1993a). Responses. Cognition and Instruction, 10(2\&3), 261-280.

diSessa, A. A. (1993b). Toward an epistemology of physics. Cognition and Instruction, 10(23), 105-225.

Duit, R. (1984). Is the second law of thermodynamics easier to understand than the first law? Tijdschrift Didactiek Natuurwetenschappen, 2(2), 102-111.

Duit, R. (1991). On the role of analogies and metaphors in learning sciences. Science Education, 75(6), 649-672.

Enghag, M., \& Niedderer, H. (2008). Two dimensions of student ownership of learning during small-group work in physics. International Journal of Science and Mathematics Education, 6(4), 629-653.

Gaggoli, R. A. (2010). Teaching elementary thermodynamics and energy conversions: Opinions. Energy, 35(2), 1047-1056.

Gentner, D. (1983). Structure-mapping: A theoretical framework for analogy. Cognitive Science, 7(2), 155-170.

Gentner, D., \& Jeziorski, M. (1993). The shift from metaphor to analogy in Western science. In A. Ortony (Ed.), Metaphor and thought (2. ed., pp. 447-480). Cambridge, UK: Cambridge University Press.

Gick, M. L., \& Holyoak, K. J. (1980). Analogical problem solving. Cognitive Psychology, 12(3), 306-355.

Glynn, S. M. (1989). The teaching with analogies model. In K. D. Muth (Ed.), Children's comprehension of text: Research into practice (pp. 185-204). Newark, DE: International Reading Association.

Granville, M. F. (1985). Student misconceptions in thermodynamics. Journal of Chemical Education, 62(10), 847-848.

Greeno, J. G. (1997). Response: On claims that answer the wrong questions. Educational Researcher, 26(1), 5-17.

Greiffenhagen, C., \& Sherman, W. (2008). Kuhn and conceptual change: on the analogy between conceptual changes in science and children. Science \& Education, 17(1), 126.

Gupta, A., Hammer, D., \& Redish, E. F. (2010). The case for dynamic models of learners' ontologies in physics. Journal of the Learning Sciences, 19(3), 285-321.

Haglund, J. (2013). Collaborative and self-generated analogies in science education. Studies in Science Education, 1-34. doi:10.1080/03057267.2013.801119

Haglund, J., \& Jeppsson, F. (2012). Using self-generated analogies in teaching of thermodynamics. Journal of Research in Science Teaching, 49(7), 898-921.

Hammer, D., \& Elby, A. (2003). Tapping epistemological resources for learning physics. Journal of the Learning Sciences, 12(1), 53-90.

Harré, R. (1959). Metaphor, model and mechanism. Proceedings of the Aristotelian Society, 60, 101-122.

Herrmann, F. (2000). The Karlsruhe Physics Course. European Journal of Physics, 21(1), 4958.

Hesse, M. B. (1966). Models and analogies in science. Notre Dame, IN: University of Notre Dame Press. 
Hestenes, D., Wells, M., \& Swackhammer, G. (1992). The force concept inventory. The Physics Teacher, 30(3), 141-158.

Heywood, D., \& Parker, J. (1997). Confronting the analogy: primary teachers exploring the usefulness in the teaching and learning of electricity. International Journal of Science Education, 19(8), 869-885.

James, M. C., \& Scharmann, L. C. (2007). Using analogies to improve the teaching performance of preservice teachers. Journal of Research in Science Teaching, 44(4), 565-585.

Jeppsson, F., Haglund, J., Amin, T. G., \& Strömdahl, H. (2013). Exploring the use of conceptual metaphors in solving problems on entropy. Journal of the Learning Sciences, 22(1), 70-120.

Jeppsson, F., Haglund, J., \& Strömdahl, H. (2011). Exploiting language in teaching of entropy. Journal of Baltic Science Education, 10(1), 27-35.

Joule, J. P. (1898). On the changes of temperature produced by the rarefaction and condensation of air. In J. S. Ames (Ed.), The free expansion of gases. Memoirs by Gay-Lussac, Joule and Joule and Thomson (pp. 15-30). New York, NY: Harper \& Brothers.

Kuhn, T. S. (1962). The structure of scientific revolutions. Chicago, IL: University of Chicago Press.

Lakatos, I. (1970). Falsification and the methodology of scientific research programmes. In I. Lakatos, \& A. Musgrave (Eds.), Criticism and the growth of knowledge (pp. 91-196). Cambridge, UK: Cambridge University Press.

Lakoff, G., \& Johnson, M. (1980). Metaphors we live by. Chicago, IL: The University of Chicago Press.

Leinonen, R., Asikainen, M. A., \& Hirvonen, P. E. (2012). University students explaining adiabatic compression of an ideal gas - A new phenomenon in introductory thermal physics. Research in Science Education, 42(6), 1165-1182.

Leinonen, R., Räsänen, E., Asikainen, M., \& Hirvonen, P. E. (2009). Students' pre-knowledge as a guideline in the teaching of introductory thermal physics at university. European Journal of Physics, 30(3), 593-604.

Lemke, J. L. (1990). Talking science. Language, learning and values. Norwood, NJ: Ablex.

Linder, C. J. (1993). A challenge to conceptual change. Science Education, 77(3), 293-300.

Loverude, M. E., Kautz, C. H., \& Heron, P. R. L. (2002). Student understanding of the first law of thermodynamics: Relating work to the adiabatic compression of an ideal gas. American Journal of Physics, 70(2), 137-148.

Mortimer, E. F. (1995). Conceptual change or conceptual profile change? Science \& Education, 4(3), 267-285.

Mozzer, N. B., \& Justi, R. (2012). Students' pre- and post-teaching analogical reasoning when they draw their analogies. International Journal of Science Education, 34(3), 429-458.

Niaz, M. (2000). A rational reconstruction of the kinetic molecular theory of gases based on history and philosophy of science and its implications for chemistry textbooks. Instructional Science, 28(1), 23-50.

Pintrich, P. R., Marx, R. W., \& Boyle, R. A. (1993). Beyond cold conceptual change: The role of motivational beliefs and classroom contextual factors in the process of conceptual change. Review of Educational Research, 63(2), 167-199.

Pittman, K. M. (1999). Student-generated analogies: Another way of knowing? Journal of Research in Science Teaching, 36(1), 1-22.

Posner, G. J., Strike, K. A., Hewson, P. W., \& Gertzog, W. A. (1982). Accommodation of a scientific conception: Toward a theory of conceptual change. Science Education, 66(2), 211-227. 
Reif, F. (1999). Thermal physics in the introductory physics course: Why and how to teach it from a unified atomic perspective. American Journal of Physics, 67(12), 1051-1062.

Schoultz, J., Säljö, R., \& Wyndhamn, J. (2001). Heavenly talk: Discourse, artifacts, and children's understanding of elementary astronomy. Human Development, 44(2-3), 103-118.

Sfard, A. (1998). On two metaphors for learning and the dangers of choosing just one. Educational Researcher, 27(2), 4-13.

Sherin, B. L. (2001). How students understand physics equations. Cognition and Instruction, 19(4), 479-541.

Smith, J. P., diSessa, A. A., \& Roschelle, J. (1993). Misconceptions reconceived: A constructivist analysis of knowledge in transition. Journal of the Learning Sciences, 3(2), 115-163.

Spiro, R. J., Feltovitch, P. J., Coulson, R. L., \& Anderson, D. K. (1989). Multiple analogies for complex concepts: Antidotes for analogy-induced misconception in advanced knowledge acquisition. In S. Vosniadou, \& A. Ortony (Eds.), Similarity and analogical reasoning. (pp. 498-531). Cambridge, UK: Cambridge University Press.

Sözbilir, M. (2001). A study on undergraduates' understandings of key chemical ideas in thermodynamics. Doctoral Dissertation, University of York, York, UK.

Tegmark, M. (1998). The interpretation of quantum mechanics: Many worlds or many words? Fortschritte der Physik, 46(6-8), 855-862.

Treagust, D. F., Harrison, A. G., Venville, G. J., \& Dagher, Z. R. (1996). Using an analogical teaching approach to engender conceptual change. International Journal of Science Education, 18(2), 213-229.

van Merriënboer, J. J. G., Shuurman, J. G., de Croock, M. B. M., \& Paas, F. G. W. C. (2002). Redirecting learners' attention during training: effects on cognitive load, transfer test performance and training efficiency. Learning and Instruction, 12(1), 11-37.

Wong, E. D. (1993). Self-generated analogies as a tool for constructing and evaluating explanations of scientific phenomena. Journal of Research in Science Teaching, 30(4), 367-380.

Wood, D., Bruner, J. S., \& Ross, G. (1976). The role of tutoring in problem solving. Journal of Child Psychology and Psychiatry, 17(2), 89-100.

Vosniadou, S., \& Brewer, W. F. (1992). Mental models of the earth: A study of conceptual change in childhood. Cognitive Psychology, 24(4), 535-585.

Yerrick, R. K., Doster, E., Nugent, J. S., Parke, H. M., \& Crawley, F. E. (2003). Social interaction and the use of analogy: An analysis of preservice teachers' talk during physics inquiry lessons. Journal of Research in Science Teaching, 40(5), 443-463.

Zook, K. B. (1991). Effects of analogical processes on learning and misrepresentation. Educational Psychology Review, 3(1), 41-72. 